\title{
Diagnóstico da geração e da composição dos RCD de Fortaleza/CE
}

\section{Generation and composition diagnosis of C\&D waste of Fortaleza/CE}

\author{
Maria Elane Dias de Oliveira \\ Mestranda do Programa de Pós-Graduação em Engenharia Civil pela Universidade Estadual de Feira de Santana (UEFS). Tecnóloga em \\ Saneamento Ambiental \\ Raquel Jucá de Moraes Sales \\ Mestranda do Programa de Pós-Graduação em Engenharia Civil (Recursos Hídricos) pela Universidade Federal do Ceará (UFC). Tecnóloga em \\ Saneamento Ambiental
}

\section{Lúcia Andréa Sindeaux de Oliveira}

Mestranda do Programa de Pós-Graduação em Economia Agrícola da UFC. Tecnóloga em Construção de Edifícios

\section{Antonio Eduardo Bezerra Cabral}

Professor do Programa de Pós-Graduação em Engenharia Estrutural e Construção Civil (PEC) da UFC. Doutor em Ciências da Engenharia Ambiental.

\section{Resumo}

Este trabalho objetivou apresentar os resultados de um diagnóstico anual da geração e da composição dos resíduos de construção e demolição de Fortaleza. Durante 12 meses, foram colhidas informações das empresas coletoras e da prefeitura, tais como a quantidade e o preço do metro cúbico do entulho coletado e o destino dado a ele. Foram ainda coletadas amostras de resíduos de construção e demolição nos locais de descarte licenciados pela prefeitura. Os resultados apontam que os locais licenciados recebem em média 702 toneladas. dia-1 ${ }^{-1}$, sendo que a composição desses resíduos de construção e demolição é, em média, de $65 \%$ de restos de argamassa, concreto e material cerâmico. Constatou-se também que grande parte desse tipo de resíduo é descartado ilegalmente, sendo que os dois principais aterros irregulares somam uma área aproximada de 26 hectares. Estima-se que esse sistema movimenta $\mathrm{R} \$ 4,5$ milhões anualmente e que quase metade desse montante é aportado somente pela Prefeitura Municipal.

Palavras-chave: RCD; diagnóstico; gestão de resíduos; Fortaleza.

\section{Abstract}

This article aimed to present the results of an annual diagnosis of generation and composition of Fortaleza's construction and demolition waste. During 12 months CDW collection companies' and the city hall' information were researched, such as the amount and the price of the cubic meter of the collected wastes and the destiny given to them. Samples of construction and demolition waste were either collected at the licensed disposal places. The results point that licensed places receive 702 tons. day ${ }^{-1}$, on average, and the composition of this construction and demolition waste is made, on average, by $65 \%$ of mortar, concrete and ceramic material. It was also verified that great part of this waste is discarded illegally, and the two main irregular disposal sites have an approximate area of 26 hectares. It is estimated that system costs approximately $\mathrm{R} \$ 4.5$ million annually and almost half of this amount is paid only by the Municipal City hall.

Keywords: CDW; diagnosis; waste management; Fortaleza. 


\section{Introdução}

A indústria da construção civil é um setor produtivo que possui considerável papel na economia do Brasil. Para tanto, segundo John (2001), esta é atualmente a maior consumidora de recursos naturais da sociedade, absorvendo de 20 a 50\% desses recursos explorados no mundo.

Como em todo processo industrial, o uso dos insumos da indústria da construção civil gera resíduos, e em grande escala, que necessitam ser gerenciados. Um ponto que demonstra a relevância dos resíduos de construção e demolição é a sua crescente participação no total dos resíduos sólidos urbanos (RSU). Na Malásia, esses resíduos correspondem, juntamente aos resíduos industriais, a 28\% do total dos RSU, enquanto que o resíduo doméstico totaliza 37\% do total (BEGUN et al., 2006). $\mathrm{Na}$ Austrália, os resíduos da indústria da construção civil correspondem a aproximadamente $37 \%$ do total de resíduos sólidos produzidos no país (TERRY, 2004). Em Hong Kong, no Kuwait e no Reino Unido, esse tipo resíduo corresponde a 38, 58 e 60\%, respectivamente (TAM e TAM, 2006; RAHAL, 2007; DHIR et al., 2004) de todo o resíduo sólido produzido, enquanto que, nos Estados Unidos, estima-se que os resíduos de construção e demolição (RCD) correspondem de 10 a 30\% do total de resíduos gerados no país (BROVIAK, 2005).

No Brasil, tem-se também sentido um crescimento na participação dos RCD no total dos RSU das cidades brasileiras. Diversas pesquisas apontam que os RCD já representam, em média, 50\% dos RSU produzidos nas cidades brasileiras, com uma taxa média de geração em torno de 0,52 tonelada.habitante ${ }^{-1}$.ano ${ }^{-1}$ (CABRAL, 2007).

A taxa de geração dos resíduos de construção e demolição é influenciada por muitos fatores; dentre eles, podem-se citar as práticas de construção e demolição adotadas, os fatores de mercado e econômicos, a estrutura reguladora que fornece incentivos para minimizar a geração de resíduos nos canteiros de obra e os desestímulos para dispor os resíduos nos aterros, entre outros fatores (BAKOSS e RAVINDRARAJAH, 1999).

A composição do RCD também é variável em função da região geográfica, da época do ano, do tipo de obra, dentre outros fatores. No Brasil, estima-se que, em média, 65\% do material descartado é de origem mineral, 13\% são madeira, $8 \%$ são plásticos e $14 \%$ são outros materiais. As construtoras são responsáveis pela geração de 20 a 25\% desse entulho, sendo que o restante provém de reformas e de obras de autoconstrução (TECHNE, 2001). Para as obras de demolição, as características de seus resíduos também variam de acordo com o tipo de estrutura a ser demolida e da técnica utilizada. Entretanto, de maneira geral, os resíduos de demolição consistem em alto percentual de material inerte, como tijolos, areia e concreto. Metais, madeira, papéis, vidro, plásticos e outros materiais também aparecem, mas em menor percentagem (POON et al., 2001).

Os custos com a gestão dos resíduos sólidos são elevados e crescentes. Entretanto, 78\% dos municípios brasileiros destinam menos de $5 \%$ dos recursos do seu orçamento para a gestão dos RSU
(BRASIL, 2002). Como exemplo, o custo para se recolher $1 \mathrm{~m}^{3}$ de RCD na cidade de São José do Rio Preto (SP) era de R\$4,90 em 1999 (PINTO, 1999), passando para até R $\$ 27,00$ em 2009 (MARQUES NETO, 2009), ou seja, um aumento de 450\% em dez anos.

Desde 2002, está em vigor a Resolução n³07 do Conselho Nacional do Meio Ambiente (CONAMA), que obriga os municípios a darem um correto destino aos RCD, impedindo-os de os descartarem em locais não licenciados, além de obrigar que o RCD do tipo A, formado basicamente por restos de concreto, argamassa, cerâmica vermelha e rochas, sejam reciclados e aproveitados como agregados ou depositados em locais previamente licenciados, para uma reciclagem futura.

Fortaleza (CE) é atualmente a quinta maior cidade do Brasil, com aproximadamente 2,5 milhões de habitantes (IBGE, 2009), e segundo seu Plano de Gestão dos Resíduos da Construção Civil (PGRCC), gera aproximadamente $3.200 \mathrm{~m}^{3}$. dia ${ }^{-1}$ de RCD (PMF, 2006). Entretanto, não se conhece quanto do RCD gerado na cidade efetivamente é gerenciado conforme as prescrições da Resolução n. ${ }^{\circ} 307$ do CONAMA, nem a composição deste. Assim, o objetivo deste trabalho foi realizar um estudo sobre o gerenciamento do RCD da cidade de Fortaleza, diagnosticando a geração e a composição do mesmo e os custos envolvidos nesse processo

\section{Metodologia}

Uma vez que a cidade de Fortaleza possui um PGRCC desde 2006, com locais de descarte e empresas prestadoras de serviço de coleta já licenciados, a quantidade de RCD gerada na cidade (aqui denominado de RCD-FOR) hipoteticamente corresponde à soma da quantidade de entulho coletada pelas empresas prestadores desse tipo de serviço (R-EMP) à quantidade coletada pela prefeitura (R-PMF), conforme Equação 1.

RCD.FOR = R.EMP + R.PMF

Equação 1

Diante disso, foram identificadas todas as empresas licenciadas que atuam no mercado de coleta do RCD da cidade de Fortaleza, utilizando-se o cadastro da Secretaria Municipal de Meio Ambiente e Controle Urbano (SEMAM) como fonte. Além disso, buscou-se tomar conhecimento dos locais licenciados pela prefeitura para o descarte desse tipo de resíduo.

Foi feito o contato com a gerência das empresas, com os órgãos da prefeitura envolvidos no gerenciamento desses resíduos e com a gerência dos locais licenciados a fim de serem coletados dados, mensalmente, por um período de 12 meses, tais como a quantidade de RCD coletada, os locais de descarte, os preços praticados pelas empresas, dentre outros.

Especificamente para o cálculo da quantidade de RCD gerada no município (RCD-FOR) foram determinados dois estimadores. $\mathrm{O}$ primeiro diz respeito à quantidade de RCD coletada pelas empresas transportadoras desse tipo de RCD, em um determinado período de 
tempo, denominado de R.EMP ( $\mathrm{m}^{3}$ de RCD.dia ${ }^{-1}$ ). Para o cálculo desse estimador, foram somadas as quantidades de RCD coletadas pelas n empresas que atuam nesse mercado (R.EMP 1, R.EMP $_{2}, \ldots$, R.EMP $_{n}$ ), dividindo o resultado pelo período em que se fez a coleta, que foi de 12 meses, conforme Equação 2.

R.EMP $=\frac{\sum_{i=1}^{n} R \cdot E M P_{i}}{12}$

Equação 2

O segundo índice corresponde à quantidade de RCD coletada pela prefeitura em um determinado período de tempo, denominado de R.PMF ( $\mathrm{m}^{3}$ de RCD.dia ${ }^{-1}$ ). Os dados da prefeitura foram também coletados por 12 meses.

Paralelamente à coleta de dados para a determinação dos índices R.EMP e R.PMF, colheram-se amostras do RCD descartado nos locais licenciados pela prefeitura. As coletas das amostras do RCD ocorreram em três momentos distintos da pesquisa, com duração de 30 dias para cada, com espaços de 3 meses entre elas.

\section{Resultados e discussão}

\section{Quantidade de RCD gerado em Fortaleza}

Foi detectado que compete à SEMAM o papel de fiscalizar e licenciar as empresas transportadoras do RCD da cidade de Fortaleza bem como os locais de descarte desses resíduos. Foram identificadas sete empresas como licenciadas pela SEMAM para realizar a coleta e o transporte do RCD de Fortaleza, aqui denominadas de empresas A, B, C, D, E, F e G. Detectou-se também que a Empresa Municipal de Manutenção e Limpeza Urbana (EMLURB) é o órgão municipal encarregado de coletar o RCD gerado pelo pequeno gerador (produtor de resíduos sólidos cujo peso específico seja maior que $500 \mathrm{~kg}$ por $\mathrm{m}^{3}$, ou cuja quantidade produzida exceda o volume de $100 \mathrm{~L}$ ou $50 \mathrm{~kg} /$ dia) e transportá-lo ao local apropriado.

Detectou-se também que Fortaleza possui duas áreas licenciadas para recebimento do RCD do tipo classe A, sendo que em uma das áreas está instalada uma usina de reciclagem, que processa os RCD transformando-os em agregados reciclados. As duas áreas são denominadas aqui de A1 e A2.

Conforme a Figura 1, que apresenta a quantidade de RCD coletada pela PMF (R.PMF) e pelas empresas privadas ao longo de 12 meses, pode-se observar que a EMLURB é a maior coletora de RCD do município, coletando em torno de 70\% do total. Dessa maneira, pode-se constatar que o pequeno gerador e o recolhimento do popularmente chamado 'entulho órfão' (entulho ao qual não se sabe a origem) geram quantidade de RCD significativamente superior ao do grande gerador.

$\mathrm{Na}$ Figura 2 tem-se o somatório da quantidade de entulho coletado pela PMF e pelas empresas privadas (RCD-FOR) e a quantidade de $\mathrm{RCD}$ recebido pelas áreas licenciadas A1 e A2. Enquanto as empresas privadas e a PMF coletaram, em média, $17.543 \mathrm{~m}^{3}$ de RCD por mês, ao longo dos 12 meses da pesquisa, os locais licenciados receberam apenas $7.660 \mathrm{~m}^{3}$ de RCD, ou seja, $43 \%$ do total. Notadamente isso demonstra que uma parte considerável do RCD coletado (57\%) não está sendo depositado nos locais licenciados.

Foi possível verificar que existe uma sazonalidade na geração desses resíduos. No período de Março a Julho de 2007 foram coletados, em média, $2.195 \mathrm{~m}^{3}$. ês $^{-1}$ de entulho pelas empresas e pela prefeitura, enquanto que, no período de Agosto a Dezembro daquele mesmo ano, a quantidade média coletada aumentou 13\%, passando a $2.482 \mathrm{~m}^{3} \cdot \mathrm{mês}^{-1}$. Esse acréscimo possivelmente ocorreu devido ao término do período de chuvas da região, que usualmente ocorrem no primeiro semestre do ano.

Considerando que o RCD possui uma massa específica de 1,2 toneladas. $\mathrm{m}^{-3}$ (IBAM, 2001), conforme os dados coletados, a PMF e as empresas privadas coletam, juntos, 702 toneladas de RCD por dia, correspondendo a uma taxa de geração de 0,12 toneladas.habitante ${ }^{-1}$. $\mathrm{ano}^{-1}$. Tal taxa de geração de RCD é baixa, quando comparada às taxas de outras cidades brasileiras e da média nacional (0,52 tonelada habitante $\mathrm{e}^{-1}$.ano $\left.{ }^{-1}\right)$ (CABRAL, 2007).

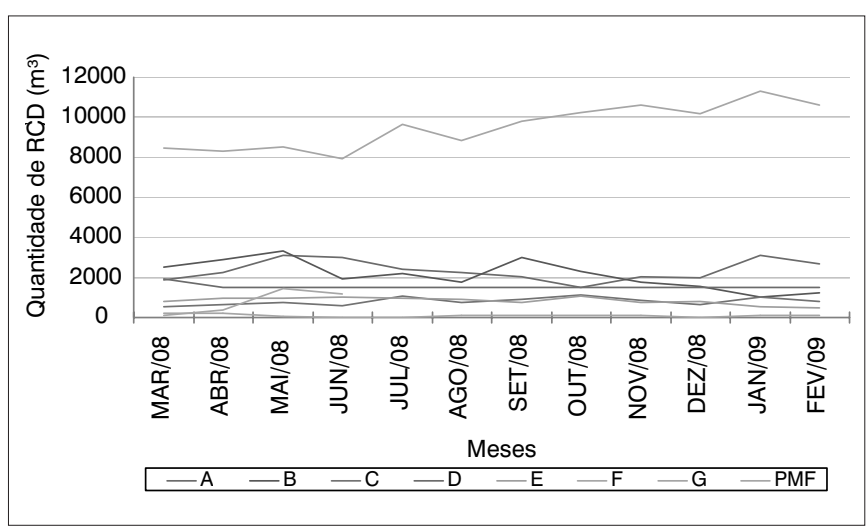

Figura 1 - Quantidade de RCD coletado pelas empresas licenciadas e pela Prefeitura Municipal de Fortaleza.

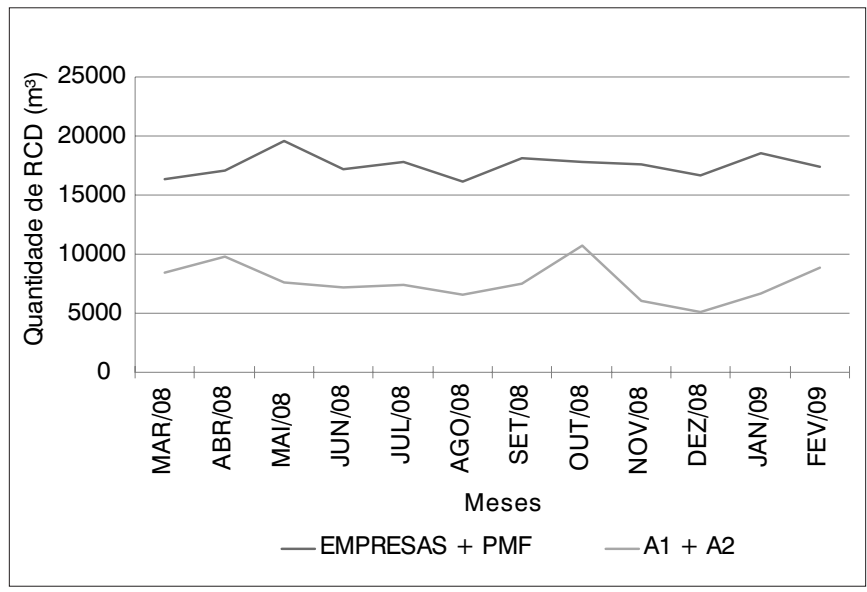

Figura 2 - Quantidade acumulada de RCD coletado pelas empresas licenciadas e pela Prefeitura Municipal de Fortaleza, e quantidade de RCD disposta nos aterros licenciados no mesmo período. 
Segundo o PGRCC da cidade, Fortaleza produz em torno de $3.200 \mathrm{~m}^{3}$ de RCD por dia, correspondendo a uma taxa de geração de 0,56 toneladas.habitante $e^{-1}$. ano ${ }^{-1}$. Caso se utilize a taxa de geração média nacional $\left(0,50\right.$ toneladas.habitante $e^{-1}$. ano $\left.{ }^{-1}\right)$, Fortaleza produziria aproximadamente 3.500 toneladas de RCD por dia.

Essa grande diferença na geração encontrada entre o que realmente é depositado nos locais licenciados e os estimadores nacional e do PGRCC deve-se à deposição feita em locais inapropriados ou irregulares, que não foram detectados por esta pesquisa.

Com o intuito de se verificar a ocorrência dessa prática em Fortaleza, buscou-se mapear os principais locais de deposição irregular de RCD na cidade. Dentre os locais de descarte irregular encontrados, detectou-se a existência de uma grande quantidade que apresentava pequenos volumes de entulho; contudo duas grandes áreas de descarte foram encontradas, conforme mostrado na Figura 3, que, somadas, aproximam-se de 26 hectares.

Conforme a Figura 3, pode-se observar que a área irregular próxima ao Estádio Governador Plácido Castelo (Castelão) possui área superior à da arena de futebol, enquanto que a outra área irregular, situada no Porto da Aldeia, está bastante próxima a uma área de preservação permanente (APP) de dunas.

O quadro descrito evidencia o desrespeito à Resolução n. ${ }^{\circ} 307$ do CONAMA e a falta de controle por parte da prefeitura municipal quanto à destinação final dos RCD do município. Dessa forma, fica evidente que, atualmente, parte do entulho gerado não tem o destino apropriado e é descartado em aterros clandestinos ou, ainda, em terrenos privados, sem o conhecimento da prefeitura.

\section{Composição do RCD gerado em Fortaleza}

Para se realizar a caracterização da composição do RCD de Fortaleza, foram coletadas amostras representativas do entulho depositado em um dos aterros licenciados. A coleta foi realizada duas vezes na semana, durante três meses, não consecutivos, ao longo dos 12 meses da pesquisa. Os materiais foram pesados separadamente, estando os resultados da caracterização das três coletas dispostas na Figura 4.

Pode-se observar que a argamassa é o principal constituinte do RCD de Fortaleza, correspondendo, em média, a 38\% da massa do RCD. Em seguida estão os resíduos de concreto e de cerâmica vermelha, com 14 e 13\%, em média, respectivamente, do total do RCD descartado. A soma do percentual desses constituintes atinge 65\% do total do RCD de Fortaleza. Esses resultados coadunam com os dos demais trabalhos desenvolvidos sobre a composição do RCD de algumas cidades brasileiras (CABRAL, 2007), uma vez que as percentagens dos constituintes argamassa, concreto e material cerâmico somam, em todas as cidades, no mínimo 62\% do total do RCD.

Esses dados confirmam a cultura construtiva brasileira, na qual as maiores perdas ocorrem nas fases de concretagem, alvenaria,

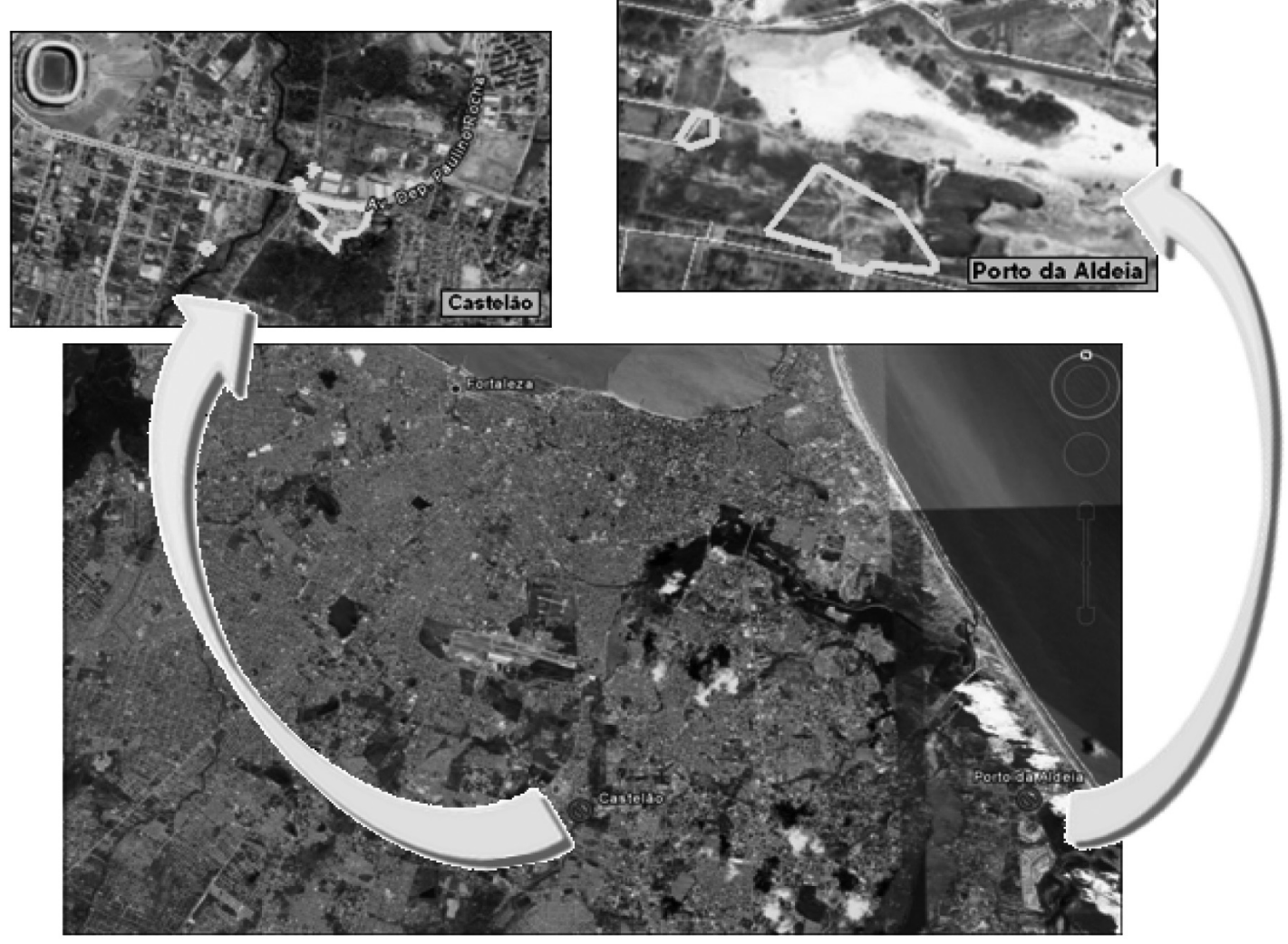

Figura 3 - Mapa de Fortaleza indicando as duas maiores áreas irregulares de descarte de RCD. 
emboço/reboco e revestimento, em que os grandes insumos utilizados são esses que aparecem em grandes quantidades nos resíduos de construção e demolição.

Ainda com relação aos dados da Figura 4, pode-se notar também a presença de uma pequena quantidade de gesso (3\% em média), disposto indevidamente, uma vez que nos aterros licenciados devese dispor somente resíduos de classe A e o gesso se enquadra nos resíduos de classe $\mathrm{C}$, conforme a resolução n. ${ }^{\circ} 307$ do CONAMA. O entulho considerado como outros, correspondendo a 3\% das amostras, diz respeito a restos de vidro, ferro, betume, folhagem, madeira, papel, plástico, tinta, alumínio, impurezas, amianto, isopor e material orgânico, que também foram encontrados nos resíduos dispostos.

\section{Custos do gerenciamento do RCD em Fortaleza}

Segundo os dados levantados no período da pesquisa, foi verificado que seis empresas trabalhavam com contêineres de capacidade média de $4,3 \mathrm{~m}^{3}$, e somente uma empresa trabalhava com caminhões basculantes, de capacidade de $12 \mathrm{~m}^{3}$. Em uma segunda verificação, detectou-se que o número de empresas que trabalhavam com caminhão basculante havia aumentado, mas o número de empresas que utilizavam os contêineres permaneceu o mesmo.

A média de preço do serviço dos caminhões basculantes em Fortaleza, no período da pesquisa, era de $\mathrm{R} \$ 20,00$ a $\mathrm{R} \$ 25,00$, ou seja seja, $\mathrm{R} \$ 1,67 \cdot \mathrm{m}^{-3}$ a $\mathrm{R} \$ 2,08 \cdot \mathrm{m}^{-3}$. No entanto, o preço praticado pelas empresas que trabalham com contêineres, em 2008, era, em média, de $\mathrm{R} \$ 70,00$ a $\mathrm{R} \$ 75,00$, ou seja, $\mathrm{R} \$ 16,28 \cdot \mathrm{m}^{-3}$ a $\mathrm{R} \$ 17,40 \cdot \mathrm{m}^{-3}$, sofrendo um aumento médio de 14\% no início de 2009.

Tais valores estão compatíveis com os encontrados por outros pesquisadores para o mesmo ano de 2009, para cidades do interior do Estado de São Paulo, como Vitória Brasil, Mirassol, São José do
Rio Preto e Catanduva, já que nessas cidades a coleta do RCD custava de $\mathrm{R} \$ 13,30 \cdot \mathrm{m}^{-3}$ a $\mathrm{R} \$ 26,67 . \mathrm{m}^{-3}$ (MARQUES NETO, 2009).

Convém salientar que os preços obtidos eram os praticados para a coleta dos resíduos classe A. Detectou-se também que os locais licenciados são remunerados para dar uma correta disposição dos RCD classe A a um valor de $\mathrm{R} \$ 8,00$ por $\mathrm{m}^{3}$ de material disposto.

Utilizando-se os valores acima, estima-se que o sistema de coleta e disposição de RCD em Fortaleza movimentou, no período da pesquisa (12 meses), aproximadamente $\mathrm{R} \$$ 4,5 milhões. Estima-se que quase metade desse montante (aproximadamente $\mathrm{R} \$ 2$ milhões) foi aportado somente pela PMF. Entretanto, convém destacar que os dados aqui apresentados referem-se somente aos números coletados e, conforme já discutido, há uma considerável parcela do entulho de Fortaleza que não foi alcançada no diagnóstico realizado neste estudo, o que induz que os custos do gerenciamento do RCD de Fortaleza são superiores aos aqui apresentados.

Várias cidades brasileiras já dispõem de usinas de reciclagem de RCD. O investimento na montagem e manutenção de usinas de reciclagem de RCD parece ser exitoso, principalmente para o setor público. Dados da usina de reciclagem Estoril, montada e mantida pela Prefeitura Municipal de Belo Horizonte (MG), apontam que os investimentos realizados haviam sido amortizados apenas nos primeiros sete meses de funcionamento, considerando-se somente a substituição de agregados naturais por reciclados e a eliminação do processo de limpeza urbana do RCD. Em um estudo recente em Fortaleza, Rios (2009) estima uma economia de R $\$ 435$ mil por ano somente na substituição dos agregados naturais da usina de asfalto da PMF pelos agregados reciclados comercializados pela usina de reciclagem de RCD privada existente no município.

Certamente os ganhos financeiros seriam bem mais extensos caso a PMF montasse e administrasse sua própria usina de reciclagem de RCD, uma vez que o uso dos agregados reciclados poderia ser expandido para

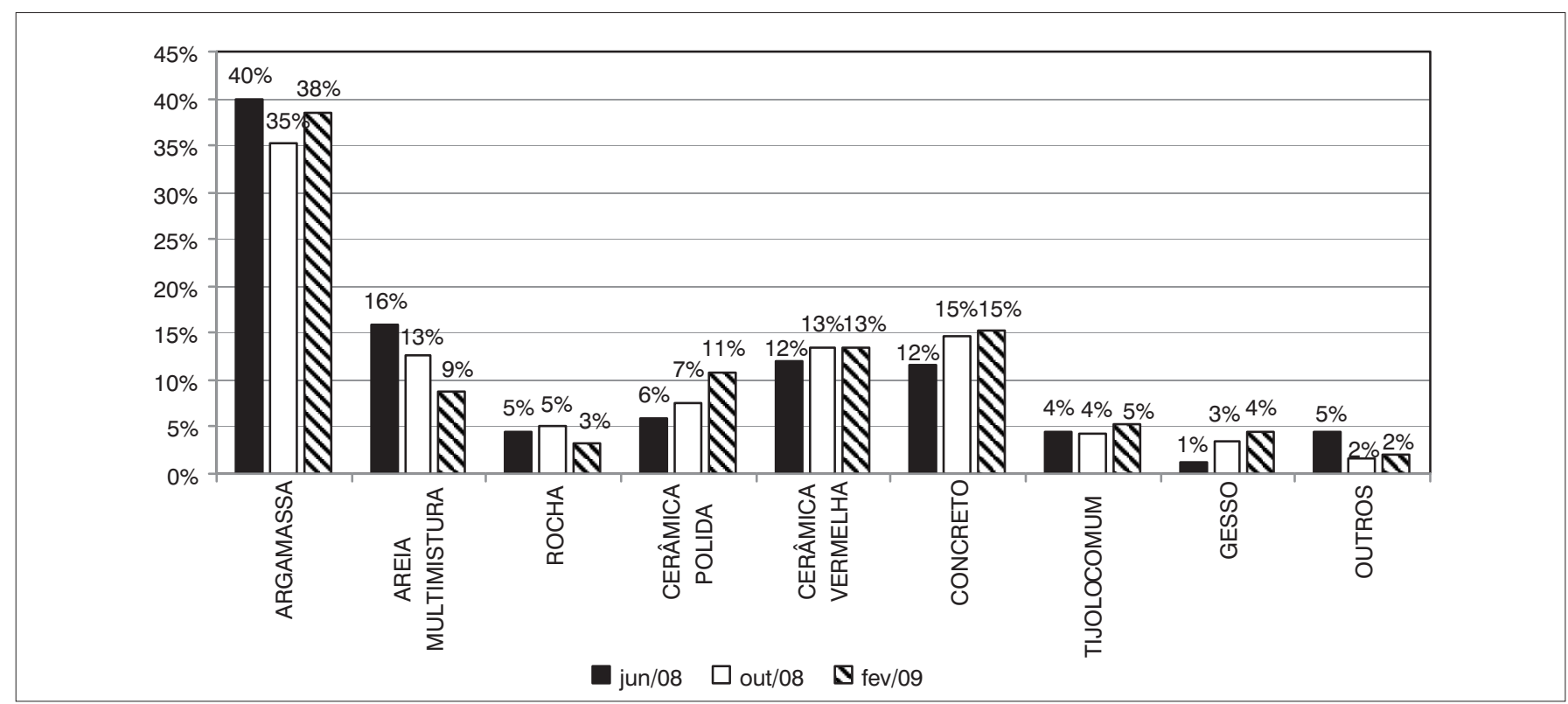

Figura 4 - Composição média do RCD coletado pelas empresas licenciadas e pela Prefeitura Municipal de Fortaleza. 
todas as obras executadas pela própria prefeitura. Fora a viabilidade financeira, com o processo de reciclagem dos RCD, a PMF daria uma aplicação clara de desenvolvimento sustentável, por meio de um aperfeiçoamento dos processos produtivos das atividades desenvolvidas pela prefeitura ligada à indústria da construção civil que utilizam o agregado como insumo.

\section{Conclusões}

Este estudo apontou que as empresas licenciadas pela SEMAM do município de Fortaleza para a coleta de RCD, juntamente da EMLURB, recolhem, aproximadamente, 706 toneladas de RCD.dia-1 ${ }^{-1}$ o que corresponde a uma taxa de geração de 0,11 toneladas de RCD.habitante-1 dia $^{-1}$. Essa taxa de geração está bem abaixo da média nacional, que é apontada de 0,5 toneladas de RCD.habitante ${ }^{-1}$. dia ${ }^{-1}$. Possivelmente, essa discrepância origina-se da não detecção, neste estudo, dos resíduos dos pequenos geradores, uma vez que se avaliou somente o resíduo transportado por empresas, que usualmente atendem somente às empresas construtoras. Usualmente os resíduos dos pequenos geradores são depositados irregularmente em locais não licenciados. Nesta pesquisa, detectaram-se dois grandes depósitos irregulares de RCD em Fortaleza. Dos constituintes do RCD de Fortaleza, a argamassa foi o principal, correspondendo, em média, a 38\% da massa do RCD. Em seguida, estiveram os resíduos de concreto e de cerâmica vermelha, com 14 e 13\%, em média, respectivamente, do total do RCD. A soma do percentual desses constituintes, os quais são passíveis de reciclagem, atinge 65\% do total do RCD de Fortaleza. Estima-se que o sistema de coleta e disposição de RCD em Fortaleza movimentou aproximadamente $\mathrm{R} \$ 4,5$ milhões nos 12 meses do estudo, dos quais aproximadamente $\mathrm{R} \$ 2$ milhões foram gastos somente pela PMF Esses valores referem-se apenas à quantidade de resíduo estimada por este estudo, sendo que há uma considerável parcela do RCD de Fortaleza que não foi aqui detectada, o que induz que os reais custos do gerenciamento desses resíduos são superiores a estes apresentados.

\section{Referências}

BAKOSS, S.L.; RAVINDRARAJAH, R.S. Recycled construction and demolition materials for use in roadworks and other local government activities. Scoping Report. 72 p. Centre for Built Infrastructure Research. University of Technology, Sydney. Sydney, 1999.

BEGUN, R.A. et al. A benefit-cost analysis on the economic feasibility of construction waste minimization: the case of Malaysia. Resources, Conservation and Recycling, v.. 48, p. 86-98, 2006.

BRASIL. Instituto Brasileiro de Geografia e Estatística (IBGE). Pesquisa nacional de saneamento básico 2000. Rio de Janeiro, 2002.

BROVIAK, P. Managing C\&D Debris. Publick Works, Febuary, p. 29-31, 2005.

CABRAL,A.E.B.Modelagem de propriedadesmecânicase de durabilidade de concretos produzidos com agregados reciclados, considerandose a variabilidade da composição do RCD. 280p. Tese (Doutorado em Ciências da Engenharia Ambiental) - Escola de Engenharia de São Carlos, Universidade de São Paulo, São Carlos, 2007.

DHIR, R.; PAINE, K.; DYER, T. Recycling construction and demolition wastes in concrete. Concrete, March, p. 25-28, 2004

INSTITUTO BRASILEIRO DE ADMINISTRAÇÃO MUNICIPAL (IBAM). Gestão integrada de resíduos sólidos: manual gerenciamento integrado de resíduos sólidos. Coordenação técnica: Victor Zular Zveibil. Rio de Janeiro, 2001.

INSTITUTO BRASILEIRO DE GEOGRAFIA E ESTATÍSTICA (IBGE). Estimativas das populações residentes, em $1^{\circ}$ de julho de 2009, segundo os municípios. Diretoria de Pesquisas - DPE. Coordenação de População e Indicadores Sociais - COPIS. 2009.

JOHN, V.M. Aproveitamento de resíduos sólidos como materiais de construção. In: CARNEIRO, A.P et al. Reciclagem de entulho para a produção de materiais de construção. Salvador: EDUFBA; 312 p.; 2001; p. 27-45.
MARQUES NETO, J.C. Estudo da gestão municipal dos resíduos de construção e demolição da bacia hidrográfica do Turvo Grande (UGRHI-15). 665 p. Tese (Doutorado em Ciências da Engenharia Ambiental) — Escola de Engenharia de São Carlos, Universidade de São Paulo, São Carlos, 2009.

PINTO, T.P. Metodologia para gestão diferenciada de resíduos sólidos da construção urbana. 203 p. Tese (Doutorado em Engenharia da Construção Civil) — Escola Politécnica da Universidade de São Paulo, São Paulo, 1999.

POON, C.S. et al. On site sorting of construction and demolition waste in Hong Kong. Resources, Conservation and Recycling, v. 32, p. 157-172, 2001.

PREFEITURA MUNICIPAL DE FORTALEZA (PMF). Plano de gerenciamento de resíduos da construção civil do município de Fortaleza-CE. 2006

RAHAL, K. Mechanical properties of concrete with recycled coarse aggregate. Building and Environmental, v. 42, p. 407-415, 2007.

RIOS, A.K.B. Os resíduos da construção civil e suas implicações socioambientais e economicas na cidade de Fortaleza. 181p. Dissertação (Mestrado em Desenvolvimento do Meio Ambiente) — Universidade Federal do Ceará, Fortaleza, 2009.

TAM, V.W.Y.; TAM, C.M. A review on the viable technology for construction waste recycling. Resources, Conservation and Recycling. v. 47, p. 209221,2006

Leal, U. Sobras que valem uma obra. TECHNE: Revista de Tecnologia da Construção, v. 10, n. 55, p. 10-14, 2001

TERRY, M. Waste minimization in the construction and demolition industry. 78p. Capstone Project (Thesis of Bachelor of Civil \& Environmental Enginnering) - Faculty of Engineering, University of Technology, Sydney, Sydney, 2004 